\title{
Satisfação dos usuários assistidos em quatro centros regionais de especialidades odontológicas do Ceará, Brasil
}

\author{
Satisfaction of users assisted in four regional dental specialties \\ centers of Ceará, Brazil
}

Ermano Batista da Costa', Joana Danielle Brandão Carneiro ${ }^{2}$, Arlete Maria Gomes Oliveira ${ }^{\mathbf{3}}$

DOI: 10.1590/0103-1104201811807

RESUMO Objetivou-se avaliar a satisfação de usuários assistidos em Centros Regionais de Especialidades Odontológicas (CEO-R) do Ceará, Brasil. Estudo analítico descritivo transversal. Foram entrevistados 518 usuários, baseado no número de atendimento por dia (136), utilizando-se como instrumento de pesquisa o Questionário de Avaliação da Qualidade dos Serviços de Saúde Bucal (QASSaB). Os dados foram analisados pelo teste Qui-quadrado de Pearson, comparando os resultados entre os CEO. Prevaleceram: sexo feminino (67,8\%), baixa escolaridade (67,8\%) e renda (63,9\%). Em relação à acessibilidade, apenas o tempo de espera na recepção $(83,5 \%)$ foi considerado positivo, com destaque desfavorável à especialidade ortodontia no tempo para obter uma vaga. As demais dimensões analisadas tiveram avaliação positiva pelo estudo. Relacionando-se as unidades de serviço pesquisadas, houve diferenças estatisticamente significantes $(p<0,05)$ na satisfação dos usuários para a maioria das dimensões analisadas, com exceção do 'auxílio de terceiros' e da 'resolutividade/satisfação com o tratamento odontológico' ( $p>0,05$ ). Concluiu-se que o nível de satisfação dos usuários participantes foi positivo, sendo a acessibilidade um problema impactante no sistema,

Universidade Estadual do Ceará (Uece) - Fortaleza (CE), Brasil.

Orcid: https://orcid. org/0000-0002-84025955

ermanobatista@hotmail.com

2 Universidade de São Paulo (USP), Faculdade de Saúde Pública (FSP) - São Paulo (SP), Brasil. Orcid: https://orcid. org/0000-0003-36298091

joanadani9@gmail.com

3 Faculdade São Leopoldo Mandic - Campinas (SP), Brasil. Universidade Estadual de Campinas (Unicamp), Faculdade de Odontologia de Piracicaba (FOP) - Campinas (SP), Brasil.

Orcid: https://orcid. org/0000-0003-3247-

2486

arlete.maria@yahoo.com.br devendo ser repensada no intuito de diminuir as eventuais insatisfações dos usuários.

PALAVRAS-CHAVE Avaliação em saúde. Saúde bucal. Atenção secundária à saúde.

ABSTRACT The objective of this research was to evaluate the satisfaction of users assisted in Regional Dental Specialties Centers (CEO-R) of Ceará, Brazil. It is a descriptive cross-sectional study. In those establishments, 518 users were interviewed, using the Oral Health Services Quality Assessment Questionnaire (QASSAB) as a research tool. The data collected were analyzed by Pearson's Chi-square test. Prevalence: female (67.8\%), low schooling (67.8\%) and income (63.9\%). Regarding accessibility issues, only the waiting time at reception (83.5\%) was considered positive, with unfavorable highlight to the specialty orthodontics in the time to obtain a vacancy. The other dimensions analyzed were positively evaluated by the study. Relating the service units surveyed, there were statistically significant differences $(p<0.05)$ in the satisfaction of users for most of the dimensions analyzed, except for the 'third-party aid' questionnaire and the 'resolution/satisfaction' with dental treatment ( $p>0.05)$. It was concluded that the level of satisfaction of the users participating in the research was generally positive, with accessibility being an impacting problem in the system, which should be rethought in order to reduce any dissatisfaction among users who require the specialized dental service of the regional network health care.

KEYWORDS Health evaluation. Oral health. Secondary care. 


\section{Introdução}

O Sistema Único de Saúde (SUS) foi criado para diminuir a fragmentação dos serviços em saúde, entre outros objetivos, e tem o constante desafio de oferecer atenção integral e continuada $^{\mathbf{1}, \mathbf{2}}$. Com a implantação da Política Nacional de Saúde Bucal (PNSB), novos princípios norteadores passaram a orientar o desenvolvimento das ações em saúde bucal na rede de atenção à saúde, com a ampliação e qualificação das Equipes de Saúde Bucal (ESB) na Estratégia Saúde da Família (ESF) ${ }^{3}$ e a partir da inserção da atenção especializada no eixo do cuidado integral em saúde bucal, com a implantação dos Centros de Especialidades Odontológicas (CEO) ${ }^{4-6}$. Os CEO foram inseridos para contribuir para a resolutividade 4 e para a integralidade do cuidado, devendo realizar, no mínimo, atividades de diagnósticos, cirurgia, periodontia, endodontia e atendimento a Portadores de Necessidades Especiais (PNE) ${ }^{7}$.

$\mathrm{Na}$ construção de indicadores nos serviços de saúde bucal brasileiros, havia carência de informações qualitativas e quantitativas, especialmente devido à ausência de uma 'cultura' institucionalizada de avaliação dos serviços, que também perpassa pela necessidade de planejamento das ações de saúde, seja no âmbito do cuidado, seja no campo da gestão; assim, o desenvolvimento do processo de avaliação passou a ser impactado ${ }^{\mathbf{8} 9}$. Estrategicamente, o Ministério da Saúde colocou a avaliação da atenção secundária em saúde bucal como prioridade $^{\mathbf{1 0}}$ e direcionou o Programa Nacional de Melhoria do Acesso e da Qualidade (PMAQ) aos CEO, que incorporaram a autoavaliação na rotina do processo de trabalho das equipes ${ }^{11,12}$. Entretanto, estudos de avaliação da atenção secundária precisam ser realizados com cautela para que possíveis vieses de seleção positiva, bem como acentuação da positividade das respostas, não aconteçam, a exemplo do que ocorreu no PMAQ Atenção Básica ${ }^{13}$.

Um importante componente passou a integrar o processo de avaliação em saúde: $o$ usuário do SUS, por intermédio do seu nível de satisfação. A avaliação centrada no usuário é evidenciada nas frustações e expectativas de utilização dos serviços necessitados, culminando, por vezes, em responsividade ou subjetividade, a qual precisa ser relativizada ${ }^{14,15}$. Contudo, o monitoramento das ações de saúde é um pilar importante para o sistema de saúde conseguir entender como a integralidade da atenção ao usuário é ofertada, sendo essencial reconhecer as necessidades da população e oferecer os recursos para respondê-las ${ }^{\mathbf{8}, 9}$. Assim, a utilização dos serviços é resultante da interação do comportamento dos usuários que procuram os cuidados e dos profissionais que os conduzem, e está intimamente relacionada aos fatores de acesso e acessibilidade ${ }^{6}$.

A compreensão da forma de organização dos serviços em saúde bucal remete à análise do contexto histórico que determinou a situação vigente, visando ao reordenamento e à qualificação do sistema9. Nesse sentido, em 1964, já existia, no estado do Ceará, o atendimento voltado para as especialidades odontológicas na capital, Fortaleza ${ }^{16}$. Antes mesmo da PNSB, o ainda denominado 'CEO Centro' começou a atender na lógica de referência ${ }^{17}$. Com o advento dos CEO Regionais (CEO-R) cearenses, todos do tipo III, a desmobilização dos centros municipais ocorrereu ${ }^{17-19}$. O estado tem adotado um modelo fundamentado em consórcios de saúde na sua política de regionalização, em que o governo custeia $40 \%$ na aquisição de equipamentos, e as vagas são distribuídas em uma Programação Pactuada Consorciada (PPC) entre os municípios e a Coordenadoria Regional da Saúde ${ }^{6,19}$.

O Ceará é um estado que busca seguir as diretrizes doutrinárias e organizativas do SUS, estruturando e qualificando sua rede de atenção à saúde, para melhor ofertar o cuidado ao usuário, razão da existência de um serviço de saúde. O estado possui $22 \mathrm{CEO}^{20}$, e os usuários que são atendidos nesses locais sempre destacam positivamente a satisfação e assistência prestada pelos profissionais para com eles. Diante desses aspectos, buscou-se avaliar 
a satisfação de usuários assistidos em Centros Regionais de Especialidades Odontológicas (CEO-R) do Ceará, a partir do estudo do grau de satisfação dos usuários assistidos, utilizando dimensões de qualidade que analisam a representatividade desses estabelecimentos, observando se existem explicações sobre as suas diferenças.

\section{Métodos}

Estudo observacional, transversal, analítico-descritivo e de natureza quantitativa foi realizado em quatro CEO-R do estado do Ceará, localizados nos municípios de Juazeiro do Norte (o maior dos CEO-R), Iguatu (o mais recente CEO-R inaugurado), Crateús (indiferente aos demais) e Ubajara (o primeiro CEO-R implantado no estado). A escolha dos respectivos centros regionais se deu de maneira intencional, por situarem-se na rota de moradia e de trabalho do pesquisador responsável pela coleta de dados.

As características dos locais são descritas para melhor entender os arcabouços do estudo. O CEO-R de Juazeiro do Norte atende aos municípios de Barbalha, Caririaçu, Granjeiro, Jardim, Missão Velha e Juazeiro do Norte, englobando uma população de 412.620 habitantes. O CEO-R Iguatu abrange uma área de 315.459 moradores de Iguatu, Acopiara, Cariús, Irapuan Pinheiro, Jucás, Mombaça, Piquet Carneiro, Quixelô e Saboeiro. O CEO-R Crateús presta serviço aos municípios de Crateús, Independência, Ararendá, Ipaporanga, Ipueiras, Monsenhor Tabosa, Nova Russas, Novo Oriente, Poranga, Quiterianópolis e Tamboril, totalizando 295.565 habitantes. O CEO-R Ubajara presta assistência a Croatá, Guaraciaba do Norte, Ibiapina, São Benedito, Ubajara e Viçosa do Ceará, abrangendo uma população de 306.828 habitantes ${ }^{20}$.

Segundo dados da Secretaria de Saúde do Estado do Ceará, os CEO-R de maior porte realizam 136 atendimentos por $\mathrm{dia}^{20}$. Assim, a estimativa da amostra foi obtida baseada nesse número de atendimentos ${ }^{21}$, por meio do seguinte direcionamento: erro amostral adotado de $2 \%$ e nível de confiança de $95 \%$, segundo a fórmula utilizada por Santos ${ }^{21}$. Para compensar as perdas, foi acrescentada à amostra 10\% de entrevistados, chegando-se a um total de 518 usuários participantes da pesquisa.

Os dados foram coletados entre outubro e dezembro de 2015, e o instrumento de pesquisa utilizado foi o Questionário de Avaliação da Qualidade dos Serviços de Saúde Bucal (Questionnaire for Quality Evaluation of Oral Health - QASSaB), validado para utilização em serviços de saúde brasileiros 22. O Questionário contempla 11 questões distribuídas em 7 dimensões: relações humanas, eficácia, acessibilidade, qualidade técnico-científica, ambiente físico/ limpeza, aceitabilidade e resolutividade. Ampliando os resultados, ocorreram acréscimos de questões versando sobre a duração do encaminhamento e sobre o auxílio de terceiros para obter a vaga, decorrente do projeto-piloto, resultando em 13 questões para o instrumento. As possibilidades de resposta ao instrumento foram construídas com base nas escalas de Likert ${ }^{23}$.

Um único pesquisador devidamente treinado participou da coleta de dados. Foram incluídos na pesquisa os usuários em tratamento nos CEO-R da pesquisa, solicitados a participar a partir da segunda consulta em diante, pois a primeira consulta é normalmente reservada aos procedimentos de diagnósticos ${ }^{20}$. Os menores de idade que concordavam com a pesquisa eram autorizados a participar por seus responsáveis, que assinaram o Termo de Consentimento Livre e Esclarecido (TCLE). Os dados foram coletados na rotina diária dos CEO, de forma a não prejudicar o funcionamento dos serviços.

Nos CEO-R do Ceará, o encaminhamento de pacientes é feito pelos cirurgiões-dentistas da atenção básica. No agendamento via comunicação entre as centrais de regulação municipais e CEO-R, são escolhidos data e horário de acordo com a disponibilidade de 
vagas, que é proporcional à população de cada município e ao valor mensal que cada município repassa ao Consórcio Público de Saúde ${ }^{20}$. Abordados na recepção, os usuários eram convocados a participar de uma pesquisa de satisfação sobre o CEO-R, sendo encaminhados a uma sala reservada, onde acontecia a entrevista, aplicando-se individualmente o instrumento de coleta de dados.

Os dados tabulados foram submetidos ao teste estatístico Qui-quadrado de Pearson. O programa estatístico usado foi o Statistical Package for the Social Sciences (SPSS) na versão 20.0, Inc., Chicago, Estados Unidos. Adotou-se o Nível de Significância de 5\% e Intervalo de Confiança (IC) de 95\%. Foram realizadas análises estatísticas com cruzamento dos dados categóricos, isolando-se a variável 'CEO' como dependente e comparando-se os resultados de cada item entre as quatro unidades diferentes, por meio das frequências das respostas dadas pelos 518 usuários. Por convenção, para considerar a dimensão avaliada como positiva ou negativa, foi adotado o escore positivo ou negativo, assim: se a frequência de resposta 5 somada a 4 resultou em pelo menos $70 \%$, foi considerada como uma avaliação positiva; caso não, negativa.

O estudo foi aprovado pelo Comitê de Ética em Pesquisa da Faculdade São Leopoldo Mandic, sob o Protocolo n ${ }^{\circ} 960.331$.

\section{Resultados}

$\mathrm{Na}$ análise descritiva da amostra, a idade dos usuários foi categorizada em quatro faixas etárias. Aquelas de até 20 anos e de 21-39 anos representaram, respectivamente, $33,1 \%$ (171) e $32,8 \%$ (170). O sexo feminino representou $67,8 \%$ (351) da população estudada; e o masculino, $32,2 \%$ (167). A maioria entrevistada, 67,8\% (351), apresentou o nível de escolaridade até o ensino fundamental, e quanto à renda, 63,9\% (339) relataram receber até um salário mínimo. Na comparação entre os CEO-R, as variáveis exibidas na tabela 1 apresentaram diferenças estatisticamente significantes $(p<0,05)$, com exceção da variável gênero.

Tabela 1. Análise descritiva da amostra de 518 usuários atendidos em Centros Regionais de Especialidades Odontológicas do Ceará; 2015

\begin{tabular}{|c|c|c|c|c|c|c|c|c|c|c|c|}
\hline \multirow{2}{*}{$\begin{array}{l}\text { Variáveis } \\
\text { independentes }\end{array}$} & \multicolumn{2}{|c|}{ CEO Juazeiro } & \multicolumn{2}{|c|}{ CEO Iguatu } & \multicolumn{2}{|c|}{ CEO Crateús } & \multicolumn{2}{|c|}{ CEO Ubajara } & \multirow[t]{2}{*}{ Valor de $\mathrm{p}$} & \multicolumn{2}{|c|}{ Grupo total } \\
\hline & $\mathbf{n}$ & $\%$ & $\mathrm{n}$ & $\%$ & $\mathrm{n}$ & $\%$ & $\mathbf{N}$ & $\%$ & & $\mathbf{N}$ & $\%$ \\
\hline \multicolumn{12}{|c|}{ Faixa etária (anos) } \\
\hline Até 20 & 58 & 39,7 & 21 & 22,3 & 54 & 38,0 & 38 & 27,9 & & 171 & 33,1 \\
\hline $21-39$ & 48 & 32,9 & 37 & 39,4 & 40 & 28,2 & 45 & 33,1 & 0,002 & 170 & 32,8 \\
\hline $40-58$ & 21 & 14,4 & 17 & 18,1 & 31 & 21,8 & 42 & 30,9 & & 111 & 21,4 \\
\hline 59 ou mais & 19 & 13,0 & 19 & 20,2 & 17 & 12,0 & 11 & 8,1 & & 66 & 12,7 \\
\hline \multicolumn{12}{|l|}{ Sexo } \\
\hline Masculino & 47 & 32,2 & 28 & 29,8 & 38 & 26,8 & 54 & 39,7 & 0,128 & 167 & 32,2 \\
\hline Feminino & 99 & 67,8 & 66 & 70,2 & 104 & 73,2 & 82 & 60,3 & & 351 & 67,8 \\
\hline \multicolumn{12}{|l|}{ Escolaridade } \\
\hline Sem instrução & 5 & 3,4 & 9 & 9,6 & 4 & 2,8 & 7 & 5,1 & & 25 & 4,8 \\
\hline Fundamental & 95 & 65,1 & 56 & 59,6 & 112 & 78,9 & 88 & 64,7 & 0,010 & 351 & 67,8 \\
\hline Médio & 35 & 24,0 & 25 & 26,6 & 23 & 16,2 & 38 & 27,9 & & 121 & 23,4 \\
\hline Superior & 11 & 7,5 & 4 & 4,2 & 3 & 2,1 & 3 & 2,3 & & 21 & 4,0 \\
\hline
\end{tabular}


Tabela 1. (cont.)

\begin{tabular}{|c|c|c|c|c|c|c|c|c|c|c|c|}
\hline \multicolumn{12}{|l|}{ Renda } \\
\hline Até 1 salário & 94 & 64,4 & 46 & 48,9 & 109 & 76,8 & 82 & 60,3 & & 331 & 63,9 \\
\hline 2 salários & 41 & 28,1 & 41 & 43,6 & 30 & 21,1 & 49 & 36,0 & 0,001 & 161 & 31,1 \\
\hline 3 salários & 8 & 5,4 & 7 & 7,5 & 2 & 1,4 & 4 & 3,0 & & 21 & 4,0 \\
\hline 4 salários ou mais & 3 & 2,1 & 0 & 0,0 & 1 & 0,7 & 1 & 0,7 & & 5 & 1,0 \\
\hline Total & 146 & 100 & 94 & 100 & 142 & 100 & 136 & 100 & & 518 & 100 \\
\hline
\end{tabular}

Fonte: Elaboração própria.

Na tabela 2, a análise se refere aos tratamentos mais procurados pelos entrevistados de acordo com as especialidades oferecidas nos CEO-R. Foram elas: Endodontia 33,8\% (175),
Ortodontia 23,5\% (122) e Prótese Dentária $22,2 \%$ (115). Salienta-se que, no período da coleta dos dados, o CEO-R Iguatu ainda não disponibilizava a especialidade Ortodontia.

Tabela 2. Análise da distribuição da amostra quanto às especialidades atendidas em Centros Regionais de Especialidades Odontológicas do Ceará; 2015

\begin{tabular}{|c|c|c|c|c|c|c|c|c|c|c|c|c|c|}
\hline \multirow{2}{*}{$\begin{array}{l}\text { CEO } \mathrm{x} \\
\text { Especialidades }\end{array}$} & \multicolumn{3}{|c|}{ CEO Juazeiro } & \multicolumn{2}{|c|}{ CEO Iguatu } & \multicolumn{2}{|c|}{ CEO Crateús } & \multicolumn{2}{|c|}{ CEO Ubajara } & \multirow[t]{2}{*}{ Valor de $p$} & \multicolumn{3}{|c|}{ Grupo total } \\
\hline & & $\mathrm{n}$ & $\%$ & $n$ & $\%$ & $\mathrm{n}$ & $\%$ & $\mathbf{N}$ & $\%$ & & & $\mathbf{N}$ & $\%$ \\
\hline Cirurgia oral & 25 & 17,1 & 17 & 18,1 & 13 & 9,2 & 20 & 14,7 & & & 75 & 14,5 & \\
\hline Endodontia & 36 & 24,7 & 47 & 50,0 & 50 & 35,2 & 42 & 30,9 & & & 175 & 33,8 & \\
\hline Ortodontia & 46 & 31,5 & 0 & 0,0 & 45 & 31,7 & 31 & 22,8 & & & 122 & 23,5 & \\
\hline Periodontia & 6 & 4,1 & 6 & 6,4 & 3 & 2,1 & 9 & 6,6 & & 0,000 & 24 & 4,6 & \\
\hline PNE & 4 & 2,7 & 0 & 0,0 & 2 & 1,4 & 1 & 0,7 & & & 7 & 1,4 & \\
\hline Prótese dentária & 29 & 19,9 & 24 & 25,5 & 29 & 20,4 & 33 & 24,3 & & & 115 & 22,2 & \\
\hline Total & 146 & 100 & 94 & 100 & 142 & 100 & 136 & 100 & & & 518 & 100 & \\
\hline
\end{tabular}

Fonte: Elaboração própria.

A tabela 3 apresenta os resultados referentes à análise quanto à procedência dos usuários. Os que residiam no município-sede dos CEO-R representaram o maior contingente de participantes, 47,5\% (246). Destacou-se o CEO-R
Juazeiro, onde $79,4 \%$ (116) eram residentes no município-sede, enquanto $76,5 \%$ (104) do CEO-R Ubajara eram advindos de municípios próximos. Já no CEO-R de Crateús, 59,9\% (85) dos entrevistados eram de lugares distantes. 
Tabela 3. Análise da amostra quanto à procedência dos usuários assistidos em Centros Regionais de Especialidades Odontológicas do Ceará; 2015

\begin{tabular}{|c|c|c|c|c|c|c|}
\hline \multicolumn{2}{|c|}{ CEO X Procedências } & $\begin{array}{r}\text { Distante }> \\
50 \mathrm{~km}\end{array}$ & $\begin{array}{r}\text { Próximo }<50 \\
\text { km }\end{array}$ & Sede & Valor de $p$ & Grupo total \\
\hline \multirow{3}{*}{$\begin{array}{l}\text { CEO } \\
\text { Juazeiro }\end{array}$} & $\mathrm{N}$ & 2 & 28 & 116 & & 146 \\
\hline & $\%$ por CEO & $1,4 \%$ & $19,2 \%$ & $79,4 \%$ & & $100,0 \%$ \\
\hline & $\%$ do Total & $0,4 \%$ & $5,4 \%$ & $22,4 \%$ & & $28,2 \%$ \\
\hline \multirow{3}{*}{$\begin{array}{l}\text { CEO } \\
\text { Iguatu }\end{array}$} & $\mathrm{N}$ & 7 & 29 & 58 & & 94 \\
\hline & $\%$ por CEO & $7,5 \%$ & $30,8 \%$ & $61,7 \%$ & & $100,0 \%$ \\
\hline & $\%$ do Total & $1,4 \%$ & $5,6 \%$ & $11,2 \%$ & & $18,2 \%$ \\
\hline \multirow{3}{*}{$\begin{array}{l}\text { CEO } \\
\text { Crateús }\end{array}$} & $\mathrm{N}$ & 85 & 9 & 48 & 0,000 & 142 \\
\hline & $\%$ por CEO & $59,9 \%$ & $6,3 \%$ & $33,8 \%$ & & $100,0 \%$ \\
\hline & $\%$ do Total & $16,4 \%$ & $1,7 \%$ & $9,3 \%$ & & $27,4 \%$ \\
\hline \multirow{3}{*}{$\begin{array}{l}\text { CEO } \\
\text { Ubajara }\end{array}$} & $\mathrm{N}$ & 8 & 104 & 24 & & 136 \\
\hline & $\%$ por CEO & $5,9 \%$ & $76,5 \%$ & $17,6 \%$ & & $100,0 \%$ \\
\hline & $\%$ do Total & $1,5 \%$ & $20,1 \%$ & $4,6 \%$ & & $26,2 \%$ \\
\hline \multirow[t]{3}{*}{ Total } & $\mathrm{N}$ & 102 & 170 & 246 & & 518 \\
\hline & $\%$ por CEO & $19,7 \%$ & $32,8 \%$ & $47,5 \%$ & & $100,0 \%$ \\
\hline & $\%$ do Total & $19,7 \%$ & $32,8 \%$ & $47,5 \%$ & & $100,0 \%$ \\
\hline
\end{tabular}

Fonte: Elaboração própria.

A tabela 4 apresenta os resultados para a avaliação da satisfação dos usuários quanto às Dimensões de Qualidade: Relações humanas, Eficácia, Qualidade técnico-científica, Ambiente físico, Resolutividade e Aceitabilidade. Os resultados foram significativos em cinco dimensões, e somente na dimensão Resolutividade não houve significância estatística quando comparados os CEO $(p>0,05)$.

Tabela 4. Avaliação da satisfação dos usuários assistidos em quatro Centros de Especialidades Regionais do Ceará, segundo as dimensões de qualidade; 2015

\begin{tabular}{|c|c|c|c|c|c|c|c|c|c|c|c|c|c|}
\hline \multirow{2}{*}{$\begin{array}{l}\text { Dimensões de } \\
\text { qualidade }\end{array}$} & \multicolumn{3}{|c|}{ CEO Juazeiro } & \multicolumn{2}{|c|}{ CEO Iguatu } & \multicolumn{2}{|c|}{ CEO Crateús } & \multicolumn{2}{|c|}{ CEO Ubajara } & \multirow[t]{2}{*}{ Valor de $p$} & \multicolumn{3}{|c|}{ Grupo total } \\
\hline & \multicolumn{2}{|r|}{$n$} & $\%$ & \multirow[t]{2}{*}{$\mathrm{n}$} & \multirow[t]{2}{*}{$\%$} & \multirow[t]{2}{*}{$n$} & \multirow[t]{2}{*}{$\%$} & \multirow[t]{2}{*}{$\mathrm{N}$} & \multirow[t]{2}{*}{$\%$} & & & \multirow[t]{2}{*}{$\mathbf{N}$} & \multirow[t]{2}{*}{$\%$} \\
\hline Relações huma & & & & & & & & & & & & & \\
\hline \multicolumn{14}{|l|}{$C D$} \\
\hline Excelente (5) & 101 & 69,2 & 64 & 68,1 & 75 & 52,8 & 80 & 58,8 & & 0,016 & 320 & 61,8 & \\
\hline Boa (4) & 45 & 30,8 & 30 & 31,9 & 67 & 47,2 & 56 & 41,2 & & & 198 & 38,2 & \\
\hline \multicolumn{14}{|l|}{ ASB } \\
\hline Excelente (5) & 100 & 68,5 & 64 & 68,1 & 76 & 53,5 & 77 & 56,6 & & 0,020 & 317 & 61,2 & \\
\hline Boa (4) & 46 & 31,5 & 30 & 31,9 & 66 & 46,5 & 59 & 43,4 & & & 201 & 38,8 & \\
\hline
\end{tabular}


Tabela 4. (cont.)

\section{Eficácia}

Não sentiu nada

61

$41,8 \quad 52$

55,3

51,4

69

50,7

$255 \quad 49,2$

(5)

Pouco desconforto 59

$40,4 \quad 41$

$43,6 \quad 60$

$42,3 \quad 50$

36,8

0,013

(4)

Desconforto (3) 20

13,7

$0,0 \quad 8$

$5,6 \quad 14$

10,3

0,0

2,2

$0,7 \quad 0$

0,0

Muito desconforto 5

3,4

1,1

$0,0 \quad 1$

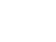

Qualidade técnico-científica

\begin{tabular}{|c|c|c|c|c|c|c|c|c|c|c|c|}
\hline Supermoderno (5) & 18 & 12,3 & 3 & 3,2 & 13 & 9,2 & 7 & 5,1 & & 41 & 7,9 \\
\hline Moderno (4) & 127 & 87,0 & 89 & 94,7 & 129 & 90,8 & 123 & 90,4 & 0,034 & 468 & 90,3 \\
\hline Mais ou menos (3) & 1 & 0,7 & 2 & 2,1 & 0 & 0,0 & 5 & 3,7 & & 8 & 1,5 \\
\hline Ultrapassado (1) & 0 & 0,0 & 0 & 0,0 & 0 & 0,0 & 1 & 0,7 & & 1 & 0,2 \\
\hline \multicolumn{12}{|c|}{ Ambiente físico/limpeza } \\
\hline \multicolumn{12}{|l|}{ Recepção } \\
\hline Excelente (5) & 103 & 70,5 & 47 & 50,0 & 70 & 49,3 & 57 & 41,9 & & 277 & 53,5 \\
\hline Boa (4) & 43 & 29,5 & 47 & 50,0 & 72 & 50,7 & 78 & 57,4 & 0,000 & 240 & 46,3 \\
\hline Regular & 0 & 0,0 & 0 & 0,0 & 0 & 0,0 & 1 & 0,7 & & 1 & 0,2 \\
\hline \multicolumn{12}{|l|}{ Banheiros } \\
\hline Excelente (5) & 76 & 52,1 & 49 & 52,1 & 64 & 45,1 & 71 & 52,2 & & 260 & 50,2 \\
\hline Boa (4) & 40 & 27,4 & 21 & 22,3 & 64 & 45,1 & 49 & 36,0 & 0,001 & 174 & 33,6 \\
\hline Não usou & 30 & 20,5 & 24 & 25,5 & 14 & 9,9 & 16 & 11,8 & & 84 & 16,2 \\
\hline \multicolumn{12}{|l|}{ Aceitabilidade } \\
\hline Sempre explica (5) & 94 & 64,4 & 72 & 76,6 & 79 & 55,6 & 80 & 58,8 & & 325 & 62,7 \\
\hline $\begin{array}{l}\text { Na maioria das } \\
\text { vezes (4) }\end{array}$ & 48 & 32,9 & 22 & 23,4 & 63 & 44,4 & 56 & 41,2 & 0,007 & 189 & 36,5 \\
\hline Não lembra (3) & 2 & 1,4 & 0 & 0,0 & 0 & 0,0 & 0 & 0,0 & & 2 & 0,4 \\
\hline Raras vezes (2) & 2 & 1,4 & 0 & 0,0 & 0 & 0,0 & 0 & 0,0 & & 2 & 0,4 \\
\hline \multicolumn{12}{|l|}{ Resolutividade } \\
\hline $\begin{array}{l}\text { Totalmente satis- } \\
\text { feito (5) }\end{array}$ & 49 & 33,6 & 29 & 30,9 & 59 & 41,5 & 56 & 41,2 & & 193 & 37,3 \\
\hline $\begin{array}{l}\text { Muito satisfeito } \\
\text { (4) }\end{array}$ & 69 & 47,3 & 50 & 53,2 & 64 & 45,1 & 49 & 36,0 & 0,101 & 232 & 44,8 \\
\hline Satisfeito (3) & 28 & 19,2 & 15 & 16,0 & 19 & 13,4 & 31 & 22,8 & & 93 & 18,0 \\
\hline Total & 146 & 100 & 94 & 100 & 142 & 100 & 136 & 100 & & 518 & 100 \\
\hline
\end{tabular}

Fonte: Elaboração própria. 
Ao analisar o modo como o CirurgiãoDentista (CD) atende (Relações humanas), 61,8\% (320) dos usuários classificaram como 'boa' a forma de atendimento; e 38,2\% (198), como 'excelente'. O CEO-R Juazeiro foi o mais bem avaliado pelos usuários nessa dimensão. Quando questionados se os cirurgiões-dentistas explicavam sobre o tratamento prestado (Aceitabilidade), 62,7\% (325) dos usuários responderam que 'sempre explicam', e 37,26\% (193) disseram que 'na maioria das vezes'. O CEO Iguatu foi o mais bem avaliado, com 76,6\% (72); e o CEO-R Crateús, o mais mal avaliado, com $55,6 \%$ (79). Cabe destacar que todos os CEO avaliados apresentaram respostas 'sempre explicam' superiores a 50\% para a dimensão Aceitabilidade.

Quanto ao incômodo no tratamento prestado (Eficácia), os resultados no grupo total apontaram satisfação positiva, cujas respostas mais frequentes foram 'pouco desconforto' 49,2\% (255) e 'não sentiu nada' - 40,5\% (210). O CEO-R Iguatu mostrou-se mais eficaz, 98,9\% (133) somando-se as respostas 'pouco desconforto' e 'não sentiu nada'. O CEO-R Juazeiro foi o menos eficaz na comparação, com 82,2\% (93) das respostas somadas para 'pouco desconforto' e 'não sentiu nada'.

Sobre a qualidade dos equipamentos (Qualidade técnico-científica), o resultado da pesquisa apontou que 90,3\% (468) dos usuários consideraram os equipamentos modernos, e apenas $0,7 \%$ (1) dos usuários mencionou que os equipamentos estavam ultrapassados. Destaque para o CEO-R Crateús, mais bem avaliado nessa dimensão, único que apresentou $100 \%$ (142) das respostas para 'supermoderno' ou 'moderno' somadas.

Sobre a estrutura da recepção (Ambiente físico/limpeza), 46,3\% (240) dos usuários consideraram 'boa'; e 53,5\% (277), 'excelente'. Destaque para os CEO-R Iguatu e Crateús que apresentaram $100 \%$ das respostas distribuídas entre as opções 'boa' ou 'excelente' na dimensão Ambiente físico/limpeza - recepção. $\mathrm{Na}$ qualidade dos banheiros, também dimensão Ambiente físico/limpeza, apenas as respostas 'bom', 33,6\% (174) e 'excelente' 50,2\% (260) foram avaliadas pelos usuários. O escore 'zero' evidenciado significou que o usuário não utilizou a estrutura dos banheiros. O CEO-R Crateús apresentou 90,2\% (128) das respostas para 'boa' ou 'excelente', sendo o mais bem avaliado. O CEO-R Iguatu apresentou a menor avaliação entre os CEO-R, com 74,4\% (70) das respostas para 'boa' ou 'excelente'.

Durante a entrevista, foi questionado se o usuário estava satisfeito com o tratamento odontológico prestado até aquele momento (Resolutividade); as respostas variaram entre 'totalmente satisfeito', 37,3\% (193), 'muito satisfeito', 44,8\% (232), e 'satisfeito', 18\% (93). O CEO-R Crateús mostrou-se mais resolutivo para a resposta 'totalmente satisfeito', 41,5\% (59); e o CEO-R Iguatu, o menos resolutivo para essa resposta, 30,9\% (29). Essa foi uma dimensão que não apresentou diferença significativa entre os CEO-R ( $p>0,05)$.

A tabela 5 mostra a avaliação da grande dimensão Acessibilidade a partir de quatro aspectos: obtenção de vaga, encaminhamento, auxílio e espera na sala. A dimensão 'espera na sala' foi a única com avaliação positiva pelos usuários, cujas respostas foram 'curto', 59,8\% (310), e 'muito curto', 23,7\% (123), para os usuários. O CEO-R Crateús foi o mais bem avaliado, com 100\% (142) das respostas distribuídas entre 'curto' ou 'muito curto'. O CEO Juazeiro foi o mais mal avaliado, com $71,9 \%$ (105) das respostas para 'curto' ou 'muito curto'. 
Tabela 5. Avaliação da satisfação dos usuários assistidos em quatro Centros Regionais de Especialidades Odontológicas do Ceará, segundo a grande dimensão acessibilidade; 2015

\begin{tabular}{|c|c|c|c|c|c|c|c|c|c|c|c|c|c|}
\hline \multirow{2}{*}{$\begin{array}{l}\text { Dimensão } \\
\text { Acessibilidade }\end{array}$} & \multicolumn{3}{|c|}{ CEO Juazeiro } & \multicolumn{2}{|c|}{ CEO Iguatu } & \multicolumn{2}{|c|}{ CEO Crateús } & \multicolumn{2}{|c|}{ CEO Ubajara } & \multirow[t]{2}{*}{ Valor de $p$} & \multicolumn{3}{|c|}{ Grupo total } \\
\hline & \multicolumn{2}{|r|}{$\mathrm{n}$} & $\%$ & \multirow[t]{2}{*}{$\mathbf{n}$} & $\%$ & \multirow[t]{2}{*}{$\mathbf{n}$} & \multirow[t]{2}{*}{$\%$} & \multirow[t]{2}{*}{$\mathbf{N}$} & \multirow[t]{2}{*}{$\%$} & & & \multirow[t]{2}{*}{$\mathbf{N}$} & \multirow{2}{*}{$\%$} \\
\hline Obtenção de Va & & & & & & & & & & & & & \\
\hline Muito fácil (5) & 19 & 13,0 & 5 & 5,3 & 39 & 27,5 & 17 & 12,5 & & & 80 & 15,4 & \\
\hline Fácil (4) & 60 & 41,1 & 74 & 78,7 & 56 & 39,4 & 70 & 51,5 & & 0,000 & 260 & 50,2 & \\
\hline $\begin{array}{l}\text { Nem fácil nem } \\
\text { difícil (3) }\end{array}$ & 47 & 32,2 & 13 & 13,8 & 26 & 18,3 & 26 & 19,1 & & & 112 & 21,6 & \\
\hline Difícil (2) & 13 & 8,9 & 0 & 0,1 & 14 & 9,9 & 7 & 5,1 & & & 34 & 6,6 & \\
\hline Muito difícil (1) & 7 & 4,8 & 2 & 2,1 & 7 & 4,9 & 16 & 11,8 & & & 32 & 6,2 & \\
\hline \multicolumn{13}{|c|}{ Encaminhamento (Semanas) } & \\
\hline Até 2 & 21 & 14,4 & 83 & 88,3 & 22 & 15,5 & 24 & 17,6 & & & 150 & 29,0 & \\
\hline 3 a 4 & 53 & 36,3 & 11 & 11,7 & 46 & 32,4 & 31 & 22,8 & & 0,000 & 141 & 27,2 & \\
\hline 5 a 8 & 23 & 15,8 & 0 & 0,0 & 29 & 20,4 & 25 & 18,4 & & & 77 & 14,9 & \\
\hline 9 a 16 & 21 & 14,4 & 0 & 0,0 & 15 & 10,6 & 22 & 16,2 & & & 58 & 11,2 & \\
\hline 17 ou mais & 28 & 19,2 & 0 & 0,0 & 30 & 21,1 & 34 & 25,0 & & & 92 & 17,8 & \\
\hline \multicolumn{14}{|l|}{ Auxílio } \\
\hline SIM & 28 & 19,2 & 20 & 21,3 & 29 & 20,4 & 17 & 12,5 & & 0,247 & 94 & 18,1 & \\
\hline NÃO & 118 & 80,8 & 74 & 78,7 & 113 & 79,6 & 119 & 87,5 & & & 424 & 81,9 & \\
\hline \multicolumn{14}{|l|}{ Espera na sala } \\
\hline Muito curto (5) & 37 & 25,3 & 7 & 7,4 & 56 & 39,4 & 23 & 16,9 & & & 123 & 23,7 & \\
\hline Curto (4) & 68 & 46,6 & 72 & 76,6 & 86 & 60,6 & 84 & 61,8 & & 0,000 & 310 & 59,8 & \\
\hline $\begin{array}{l}\text { Nem longo nem } \\
\text { curto (3) }\end{array}$ & 32 & 21,9 & 15 & 16,0 & 0 & 0,0 & 24 & 17,6 & & & 71 & 13,7 & \\
\hline Longo (2) & 8 & 5,5 & 0 & 0,0 & 0 & 0,0 & 4 & 2,9 & & & 12 & 2,3 & \\
\hline Muito longo (1) & 1 & 0,7 & 0 & 0,0 & 0 & 0,0 & 1 & 0,7 & & & 2 & 0,4 & \\
\hline Total & 146 & 100 & 94 & 100 & 142 & 100 & 136 & 100 & & & 518 & 100 & \\
\hline
\end{tabular}

Fonte: Elaboração própria.

Para a dimensão 'obtenção de vaga' nos CEO-R, os usuários mencionaram o grau de dificuldade desde 'muito fácil', 15,24\% (80), até 'muito difícil', 6,2\% (32), na amostra total. A maior dificuldade, segundo os usuários, para obtenção da vaga está no CEO-R Ubajara, pois as respostas 'difícil' ou 'muito difícil', 16,9\% (23), foram maiores em relação aos outros CEO-R: 14,8\% (Crateús), 13,7\% (Juazeiro) e 2,3\% (Iguatu). A menor dificuldade, ou seja, onde a obtenção da vaga é mais 'fácil' ou 'muito fácil', está no CEO-R Iguatu,
84\% (79), comparado aos outros CEO-R: 66,9\% (Crateús), 64\% (Ubajara) e 54,1\% (Juazeiro).

Para a dimensão 'encaminhamento', o CEO-R Iguatu foi o mais bem avaliado em relação aos outros, haja vista que apresentou menor tempo de espera, até 2 semanas, para 88,3\% (83) dos usuários. Para os CEO-R Juazeiro e Crateús, a maioria dos usuários, $36,3 \%$ (53) e $32,4 \%$ (46), respectivamente, respondeu que o tempo de espera varia de 3 a 4 semanas. O CEO-R Ubajara apresentou o 
pior resultado nesse aspecto entre todos os CEO-R avaliados, com 25\% (34) dos usuários informando que o tempo de espera pode levar 17 semanas ou mais.

A dimensão 'Auxílio' questionava se os usuários tiveram auxílio de terceiros para obtenção da vaga no CEO-R. Dos usuários, 81,9\% (424) responderam 'não'. Esta foi uma dimensão que não apresentou diferença significativa entre os CEO ( $>>0,05)$.

\section{Discussão}

Os CEO-R pesquisados estão localizados em regiões político-geográficas diferentes, no estado do Ceará (Crateús - Sertão; Juazeiro - Cariri; Iguatu - Zona de Transição Sertão/ Cariri; e Ubajara - Serra da Ibiapaba), o que ampliou diferentes olhares. Esses estabelecimentos de saúde possuem gestão autônoma, resultantes da mesma política de saúde implantada pelo anterior Governo do Estado do Ceará no período 2007 a $2014^{20}$.

Os resultados da pesquisa apontaram uma parcela de usuários com baixa situação socioeconômica, corroborando outros estudos 22,24,25. Denota-se o papel dos CEO em garantir o princípio constitucional da equidade e acrescenta-se que os serviços prestados neles vão ao encontro das políticas sociais vigentes, as quais consideram a vulnerabilidade dos indivíduos, refletidas inclusive nas condições de saúde bucal.

Nesta pesquisa, a maior representação foi do sexo feminino, que provavelmente se explica pela maior disciplina das mulheres em relação ao cuidado à saúde e por terem mais tempo disponível, pois, muitas vezes, são cuidadoras do lar' ${ }^{26}$. Tal fato pode limitar a interpretação de alguns dados por atuar como fator de conclusão?.

Outra variável bem representada foi em relação à faixa etária, refletido pelo usuário considerado 'jovem', e à especialidade endodontia, como sendo predominante no estudo. Uma possível relação desses fatores é o fato de as pessoas jovens serem atendidas mais em especialidades como a endodontia, e tal atendimento vai diminuindo consideravelmente nas idades avançadas ${ }^{27}$, tendo em vista que a população adulta e idosa, especialmente esta última, apresenta índices elevados de edentulismo, conforme aponta o levantamento epidemiológico de Saúde Bucal, SB Brasil 201028. Contudo, observa-se a necessidade de maior efetividade na marcação em endodontia, pois a demora no atendimento pode levar à mutilação dentária $^{29}$. Salienta-se ainda a necessidade de uma política de saúde bucal que também integre o trabalhador na sua dimensão do cuidado, pois este, muitas vezes, acaba optando por atendimento imediato (mais 'rápido') como a exodontia ${ }^{30}$, contribuindo para o aumento do número de desdentados na população, além da diminuição ou perda de funções primordiais do sistema estomatognático (mastigar, sorrir).

Na presente pesquisa, os dados socioeconômico-geográficos, em geral, apresentaram diferenças estatísticas significantes $(\mathrm{p}<0,05)$, com exceção da questão do sexo. Os resultados não mostraram diferenças estatísticas significantes $(p>0,05)$ na comparação entre os CEO, no que diz respeito à dimensão resolutividade, nem na questão auxílio de terceiros para o usuário obter a vaga no CEO. Isso denota padrão semelhante de organização nos CEO no quesito tratamento prestado, e que o auxílio para conseguir uma vaga ocorre da mesma forma nos centros odontológicos. Os CEO-R cearenses estudados mostraram semelhanças em seus aspectos estruturais, humanos e operacionais ${ }^{\mathbf{1 6}}$, diferentemente, por exemplo, dos CEO do município de Natal, no Rio Grande do Norte, que apresentaram distinções entre $\mathbf{s i}^{\mathbf{1 4}}$. É fato que os processos de implantação, construção e consolidação do SUS devem ser subsidiados pela análise de medidas demográficas, epidemiológicas e de indicadores de serviços de saúde ${ }^{31}$, o que pode justificar tais distinções especialmente se a equidade não for um 
princípio considerado na instituição das políticas sociais e de saúde.

Referente à procedência dos usuários nos CEO-R de Juazeiro do Norte e Iguatu, em sua maioria, eram provenientes da sede do próprio município ${ }^{14}$, podendo justificar-se pelo maior aporte populacional ${ }^{32}$ e pelo maior montante de recursos destinados ao Consórcio Público de Saúde, tendo direito a mais vagas ${ }^{20}$. Dos entrevistados no CEO-R Crateús, mais da metade era proveniente de municípios distantes da sede, por outro lado, os entrevistados no CEO-R Ubajara eram, em sua maioria, de municípios próximos à sede do CEO. Logo, a distância pode não ter influência na acessibilidade aos CEO, pois a diferença locorregional pareceu mais importante.

No presente estudo, os usuários mostraram-se bastantes satisfeitos, tanto com o atendimento do cirurgião-dentista como do auxiliar de saúde bucal, bem como demonstraram satisfação com as explicações dadas pelo cirurgião-dentista no ato da consulta ${ }^{25}$. $\mathrm{O}$ acolhimento adequado inicia-se com boas ações comunicacionais já na recepção dos serviços, seguido por princípios de humanização, como o respeito, a cordialidade e o direito de ser esclarecido sobre os passos e sobre os procedimentos dos exames ${ }^{33-35}$.

A avaliação positiva da estrutura física da recepção dos CEO pode relacionar-se com atrativos observados, como: TV, água para beber e ar-condicionado, ambiente limpo, espaços adequados, entre outros ${ }^{20}$. No ato da entrevista, evidenciou-se que os usuários estendiam seus bons comentários à força de trabalho da recepção (recepcionista e vigia), o que se confirmou quando $53,5 \%$ consideraram como excelente a estrutura da recepção, contrapondo-se aos resultados da pesquisa de Marques $^{36}$ em que se indicou preocupação com o funcionamento desse setor.

Os resultados da avaliação dos equipamentos considerados modernos pela maioria dos usuários do estudo (90,3\%) foram semelhantes e distintos aos de outras pesquisas ${ }^{\mathbf{1 6}, 26}$. As características socioeconômicas e a percepção do usuário podem influenciar na sua satisfação com os serviços de saúde ${ }^{37}$, especialmente se este for um serviço que alcança uma parcela reduzida de usuários e/ou abrigue especialidades que tenham um caráter restritivo ou elitista.

Na obtenção de vaga, 50,2\% dos usuários declararam ser fácil conseguir uma vaga no CEO. Apesar disso, conforme padronização do estudo, essa questão teve avaliação negativa. Usando critérios diferentes dos realizados nessa pesquisa, estudo realizado nos CEO de Recife mostrou que $52,5 \%$ dos usuários também manifestaram avaliações negativas quando relacionadas com a obtenção de vagas nos $\mathrm{CEO}^{38}$. Observou-se, neste estudo, que alguns usuários não passaram pela regulação da Central de Marcação de Consultas de seus municípios (18,1\%), tendo o acesso à vaga no CEO facilitado por terceiros (gestores e servidores municipais, vereadores e até funcionário ou profissional de saúde do CEO), condição denominada 'auxílio'38. Há relatos deste atalho para o acesso direto aos CEO em várias localidades brasileiras ${ }^{4}$, o que acaba descaracterizando a rede de atenção e gerando dupla porta de acesso ao SUS. A gestão pública, bem como os atores sociais que a fazem acontecer, devem prezar por práticas que evitem duplicidades dos serviços de saúde e promover inclusão, cidadania e justiça social ${ }^{39}$. Desse modo, uma característica que influencia substancialmente a efetivação da rede em saúde bucal consiste no pouco conhecimento do sistema pelo usuário e, muitas vezes, pelos próprios profissionais, especialmente sobre os pontos de atendimento, os fluxos e os limites de atuação de cada nível de atenção ${ }^{40}$.

Referente ao tempo para o usuário ser atendido na sala de espera dos CEO, a avaliação positiva $(83,5 \%)$ na satisfação pelo usuário pode ser devido à boa organização da demanda, o que diminui consideravelmente a expectativa dele pelo atendimento. Alguns estudos ${ }^{16,26}$ corroboraram esses 
achados, os quais apontaram que a maioria dos usuários considerou baixo o tempo de espera na recepção para o atendimento. Quando os serviços de saúde atendem por ordem de chegada, levam os usuários a se acostumar com a espera pelo atendimento, independentemente da hora marcada ${ }^{37}$, isto pode ser um contraponto, considerando-se os critérios distintos para o atendimento nos CEO pesquisados.

$\mathrm{Na}$ associação das especialidades ao tempo de espera por uma vaga no CEO, observou-se maior tempo de espera na especialidade ortodontia, embora em outros estudos apenas a endodontia apresente-se como demanda reprimida ${ }^{\mathbf{2 4} 26}$. Percebeu-se menor espera para a especialidade periodontia e portadores de necessidades especiais, corroborando o estudo de Casotti28. É certo que a oferta das especialidades odontológicas deve ser ampliada conforme análise locorregional ${ }^{7}$ das necessidades de saúde.

Referente ao acesso, quando comparado aos demais CEO estudados, o de Iguatu recebeu a melhor avaliação, visto que $78,7 \%$ consideraram fácil a obtenção da vaga, e $88,3 \%$ esperaram apenas 2 semanas para concluir o encaminhamento. Tais resultados favoráveis podem estar relacionados com o fato desse Centro ter sido reinaugurado $20 \mathrm{em}$ junho de 2014, não apresentando demandas reprimidas de anos anteriores. É importante o despertar da gestão dos CEO-R, no sentido de buscar estratégias para controlar o número de usuários que sofrem com dor, tanto física como psicológica, devido à grande espera por uma vaga. Devem ainda entender o problema da acessibilidade na raiz de sua implantação; e, no Consórcio Público de Saúde, enfrentar o desafio da questão.

O perfil de baixa renda da população, representado nesta pesquisa, pode ter facilitado à situação do gratitude bias, a sensação de gratidão pelo tratamento gratuito ${ }^{\mathbf{4 1}}$, visto que, mesmo diante das dificuldades perante o encaminhamento até a realização do tratamento, muitos se consideraram satisfeitos com os serviços. É inegável o papel crítico que os usuários desempenham nas pesquisas de satisfação, mas a possibilidade da aceitação passiva do serviço deve ser considerada ${ }^{15}$.

Sugere-se que mais estudos devam ser realizados, apresentando fontes de outras localidades, fornecendo, dessa forma, informações estatísticas que, somadas, mostrem a realidade dos serviços de saúde bucal do estado do Ceará, auxiliando na formulação de estratégias que atuem nos motivos de insatisfação dos usuários. Outro ponto importante a ser avaliado são as centrais de marcação de consultas nos municípios, de forma a desarticular os possíveis privilégios que facilitam desigualmente a obtenção de vaga nos $\mathrm{CEO}$.

Investigar problemas que porventura aconteçam em nível municipal, como a falta ou rotatividade de cirurgiões-dentistas ${ }^{42}$ e a disposição destes para encaminhar os pacientes, o que pode dificultar a formação de vínculo profissional-usuário, merece atenção. Igualmente, a contrapartida e o interesse das administrações municipais com a causa da saúde bucal devem ser revistos. Sugere-se ainda investigar o impacto do incômodo nas especialidades oferecidas isoladamente e ainda saber as principais manobras dos profissionais para amenizar o desconforto com os procedimentos odontológicos.

Este estudo revela à comunidade científica parte da estrutura funcional e do processo de trabalho de CEO-R do Ceará, na visão dos usuários assistidos no serviço, servindo de parâmetro para o planejamento de novas ações que melhorem as condições de saúde bucal e de vida da população usuária de estabelecimentos públicos de saúde. Entretanto, ele limita-se por ouvir apenas um 'elo' que constrói o serviço de saúde bucal do estado do Ceará. Estudos que considerem a visão de profissionais e gestores podem ajudar a ampliar o olhar sobre a dimensão acessibilidade e, possivelmente, contribuir para melhor oferta da integralidade do cuidado em saúde bucal. 


\section{Conclusões}

O acesso aos CEO-R, no Ceará, representou o nó crítico para a insatisfação entre os usuários do serviço na dimensão Acessibilidade para 'obtenção de vaga' e 'encaminhamento'. O elevado tempo de espera para obter uma vaga merece atenção, sendo o auxílio de terceiros, algumas vezes, uma via para driblar essa barreira.

\section{Colaboradores}

Oliveira AMG contribuiu para a elaboração com as seguintes atividades: concepção e planejamento para análise e interpretação dos dados, revisão crítica do conteúdo e participação da aprovação da versão final do artigo. Carneiro JDB contribuiu para a elaboração com as seguintes atividades: interpretação dos dados, revisão crítica do conteúdo e participação da aprovação da versão final do artigo. Costa EB contribuiu para a elaboração com as seguintes atividades: concepção e planejamento ou análise e a interpretação dos dados; aprovação da versão final do manuscrito.

\section{Referências}

1. Spedo SM, Pinto NRS, Tanaka OY. O difícil acesso a serviços de média complexidade do SUS: o caso da cidade de São Paulo, Brasil. Physis. 2010; 20(3):95372.

2. Contarato PC. Política nacional e contexto local: uma análise da implementação da política de saúde bucal no Município de Vitória, do Espírito Santo [dissertação]. Rio de Janeiro: Fiocruz/Ensp; 2011.

3. Brasil. Ministério da Saúde. Secretaria de Atenção à Saúde. Departamento de Atenção Básica. Diretrizes da Política Nacional de Saúde Bucal. Brasília, DF: MS; 2004

4. Narvai PC. Avanços e desafios da Política Nacional de Saúde Bucal no Brasil. Tempus. 2009; 5(3):21-34.

5.

Frazão P, Narvai PC. Saúde bucal no Sistema Único de Saúde: 20 anos de lutas por uma política pública. Saúde debate. 2009; 33(81):64-71.

6. Pinto VPT, Teixeira AH, Santos PR, et al. Avaliação da acessibilidade ao Centro de Especialidades Odontológicas de abrangência macrorregional de Sobral, Ceará, Brasil. Ciênc Saúde Colet. 2014; 19(7):2235-2244.

7. Brasil. Ministério da Saúde. Secretaria de Atenção à Saúde. Departamento de Atenção Básica. Saúde bucal: caderno de atenção básica. Brasília, DF: MS; 2006.

8. Goes PSA, Figueiredo N, Neves JC, et al. Avaliação da atenção secundária em saúde bucal: uma investigação nos centros de especialidades do Brasil. Cad Saúde Pública. 2012; 28:81-89. 
9. Castro RD. Avaliação em saúde bucal na perspectiva do usuário: enfoque ao estudo da acessibilidade organizacional [dissertação]. Natal: UFRN; 2007.

10. Brasil. Ministério da Saúde. Secretaria de Atenção à Saúde. Departamento de Atenção Básica. Manual de especialidades em saúde bucal. Brasília, DF: MS; 2008.

11. Brasil. Ministério da Saúde. Secretaria de Atenção à Saúde. Departamento de Atenção Básica. Programa nacional de melhoria do acesso e da qualidade da atenção básica (PMAQ-AB): manual instrutivo. Brasília, DF: MS; 2013.

12. Linhares PHA, Lira GV, Albuquerque IMN. Avaliação do Programa de Melhoria do Acesso e da Qualidade no estado do Ceará. Saúde debate. 2014; 38:195-208.

13. Fausto MCR, Giovanella L, Mendonça MHM, et al. A posição da Estratégia Saúde da Família na rede de atenção à saúde na perspectiva das equipes e usuários participantes do PMAQ-AB. Saúde debate. 2014; 38:13-33.

14. Souza GCA. Centro de Especialidades Odontológicas: avaliação da atenção de média complexidade na rede pública da Grande Natal [dissertação]. Natal: UFRN; 2009.

15. Hollanda E, Siqueira SAV, Andrade GRB, et al. Satisfação e responsividade em serviços de atenção à saúde da Fundação Oswaldo Cruz. Ciênc Saúde Colet. 2012; 17(12):3343-3352.

16. Bezerril DO. Resolutividade de Centros de Especializadas Odontológicas Regionais do Estado do Ceará [dissertação]. Fortaleza: Unifor; 2011.

17. Guimarães MLF. Cuidados Secundários em Saúde Bucal: Centro de Especialidades Odontológicas no Ceará, Brasil [dissertação]. Fortaleza: Uece; 2008.

18. Marques PLP. Instrumento de informação para controle, avaliação, monitoramento e auditoria: a experiência do CEO Regional de Baturité [monografia].
Fortaleza: Universidade Vale do Acaraú; 2010.

19. Filgueira ACC. Avaliação de desempenho da política pública dos Centros de Especialidades Odontológicas na região do Cariri - CE [dissertação]. Fortaleza: UFC; 2012.

20. Ceará (Estado). Governo do Estado [internet]. Fortaleza: Governo do Estado; 2016 [acesso em 2016 ago 5]. Disponível em: http://www.ceara.gov.br.

21. Santos GEO. Cálculo amostral: calculadora on-line [intetnet]. [acesso em 2014 jun 12]. Disponível em: http://www.publicacoesdeturismo.com.br/calculoamostral/.

22. Kitamura ES, Bastos SS, Palma PV, et al. Avaliação da satisfação dos usuários dos Centros de Especialidades Odontológicas da macrorregião Sudeste de Minas Gerais, 2013. Epidemiol Serv Saúde. 2016; 25(1):137-148.

23. Backer P. Gestão ambiental: a administração verde. Rio de Janeiro: Qualitymark; 2005.

24. Deitos AR. Avaliação na atenção especializada em saúde bucal [dissertação]. São Paulo: USP; 2009.

25. Magalhães BG, Oliveira RS, Góes PSA, et al. Avaliação da qualidade dos serviços prestados pelos Centros de Especialidades Odontológicas: visão dos usuários. Cad Saúde Colet. 2015; 23(1):76-85.

26. Andrade KLC, Ferreira EF. Avaliação da inserção da Odontologia no Programa Saúde da Família de Pompéu (MG): a satisfação do usuário. Ciênc Saúde Colet. 2006; 11(1):123-130.

27. Casotti E, Contarato PC, Fonseca ABM, et al. Atenção em Saúde Bucal no Brasil: uma análise a partir da Avaliação Externa do PMAQ-AB. Saúde debate. 2014; 38(esp):140-157.

28. Brasil. Ministério da Saúde. Pesquisa Nacional de Saúde Bucal: Resultados Principais. Brasília, DF: MS; 2012. 
29. Santos AM, Assis MMA. Da fragmentação à integralidade: construindo e (des)construindo a prática de saúde bucal no Programa de Saúde da Família (PSF) de Alagoinhas, BA. Ciênc Saúde Colet. 2006; 11(1):53-61.

30. Ferraz MAAL. Avaliação da referência e contra-referência nos Centros de Especialidades Odontológicas em Teresina-Piauí [dissertação]. Teresina: Universidade Federal do Piauí; 2008.

31. Rode SM, Nunes SG. Atualização clínica em odontologia. São Paulo: Artes Médicas; 2004.

32. Instituto Brasileiro de Geografia e Estatística. Um panorama da saúde no Brasil: acesso e utili-zação de serviços, condições de saúde e fatores de risco e proteção à saúde, 2008. Rio de Janeiro: IBGE; 2010.

33. Assis MMA, Jesus WLA. Acesso aos serviços de saúde: abordagens, conceitos, políticas e modelo de análise. Ciênc Saúde Colet. 2012; 17(11):2865-2875.

34. Costa ICC. Planejamento das ações de saúde. In: Ferreira MAF, Roncalli AG, Lima KC, organizadores. Saúde bucal coletiva: conhecer para atuar. Natal: UFRN; 2005. p. 287-300.

35. Silvestre JAC. Coordenação das ações de atenção à saúde bucal na 13a coordenadoria regional de saúde do estado do Ceará: percepção da gestão municipal [dissertação]. Sobral: UFC; 2012.

36. Marques PLP, Freitas KM, Saintrain MVL, et al. Análise do funcionamento de um Centro de Especialidades Odontológicas na perspectiva dos usuários. Convibra saúde. 2013; 1:105.
37. Lima ACS, Cabral ED, Vasconcelos MMVB. Satisfação dos usuários assistidos nos Centros de Especialidades Odontológicas do Município do Recife, Pernambuco, Brasil. Cad Saúde Pública. 2010; 26:991-1002.

38. Gouveia GC, Souza WV, Luna CF, et al. Health care users satisfaction in Brazil, 2003. Cad Saúde Pública. 2005; 21(supl.1):S109-S118.

39. Lucchese PTR. Equidade na gestão descentralizadado SUS: desafios para redução de desigualdades. Ciênc Saúde Colet. 2003; 8(2):439-448.

40. Mello ALSF, Andrade SR, Moyses SJ, et al. Saúde bucal na rede de atenção e processo de regionalização. Ciênc Saúde Colet. 2014; 19(1):205-214.

41. Travesso-Yépez M, Morais NA. Reivindicando a subjetividade dos usuários da Rede Básica de Saúde: para humanização do atendimento. Cad Saúde Pública. 2004; 20:80-88.

42. Costa PM, Medeiros Júnior A, Costa ICC, et al. Processo de trabalho do dentista na Estratégia de Saúde da Família do município de Parnamirim-RN: enfrentando os desafios de um novo modelo de atenção. Rev Odontol Bras Central. 2010; 19(51):327332.

Recebido em 21/03/2018

Aprovado em 11/09/2018

Conflito de interesses: inexistente

Suporte financeiro: não houve 